[Supporting Information]

\title{
Concurrent Imaging of Surface-Enhanced Raman and Mie Scattering from Built-in Nanogap Plasmonic Particles
}

Sunghee Lee ${ }^{\mathrm{a} \uparrow}$, Jungwoo Kim ${ }^{\mathrm{b}, \mathrm{c}_{\dagger}}$, Kyungnae Baek ${ }^{\mathrm{a}}$, Nam Hoon Kim ${ }^{\mathrm{d}}$, Jerome K. Hyun ${ }^{\mathrm{a}^{*}}$, So-Jung Park ${ }^{\mathrm{a}^{*}}$ and Haemi Lee $\mathrm{b}^{\mathrm{*}}$

a Department of Chemistry and Nanoscience, Ewha Womans University, 52 Ewhayeodae-gil, Seodaemun-gu, Seoul 03760, Korea

${ }^{\mathrm{b}}$ Therapeutics and Biotechnology Division, Korea Research Institute of Chemical Technology (KRICT), 141 Gajeong-ro, Yuseong-gu, Daejeon 34114, Korea

${ }^{\mathrm{c}}$ Center for National R\&D Budget Strategy, Korea Institute of Science \& Technology Evaluation and Planning (KISTEP), 1339 Wonjung-ro, Maengdong-myeon, Eumseong-gun, Chungcheongbuk-do, 27740, Korea

${ }^{\mathrm{d}}$ Center for Convergent research of Emerging Virus Infection, Korea Research Institute of Chemical Technology (KRICT), 141 Gajeong-ro, Yuseong-gu, Daejeon 34114, Korea

*Corresponding Authors: sojungpark@ewha.ac.kr (So-Jung Park), haemilee@krict.re.kr (Haemi Lee), kadam.hyun@ewha.ac.kr (Jerome K. Hyun)

$\dagger$ These authors contributed equally. 


\section{Experimental Section}

Working principle of bimodal imaging assembly. As schematically presented in Figure 1b, a 660-nm incident source was selected to simultaneously excite SERS and Mie scattering signals from individual raspberry-MMs. A wide-field illumination area at the sample surface was achieved using a beam expander $(4 \times \mathrm{BE})$ consisting of two lenses with a 1:4 focal length ratio. Lens 1 with a focal length of $500 \mathrm{~mm}$ was used to focus the incident light onto the back focal plane of the objective $(\times 100,1.49$ N. A.). A broadband beamsplitter (Chroma Technology Corp., VT) with $20 \%$ transmittance was inserted instead of the dichroic mirror placed originally inside the IX-71 microscope (Olympus, Japan). The target signal collected by the objective was passed through a tube lens (Lens 2) with a focal length of $175 \mathrm{~mm}$. In order to collect the target signals (dark yellow) without the incident background (purplish pink), we used Lens 3 with a focal length of $185 \mathrm{~mm}$ to ensure the lateral position of the background light was sufficiently separated from that of the target signal. The target signal was then spatially filtered by positioning the slit at its focal plane $\mathbf{d}$, whereas the background focused at plane $\mathbf{c}$ was directed off the detection path. The target signal was collimated by Lens 4 and then split into the inelastic (upper) and elastic (lower) channels using two dichroic mirrors $\left(1^{\text {st }} \mathrm{DC}, 2^{\text {nd }}\right.$ DC). Technically, for multiplexed imaging, an identical pair of dichroic mirrors can be used to switch between two excitation wavelengths $(532$ and $660 \mathrm{~nm})$ without fixing the detection path. Lenses 5 and 6 were used to focus the inelastic and elastic signals onto the image plane of the EM CCD, respectively. A narrow band-pass filter (NB Filter) and long-pass filter (LP Filter) were used in the inelastic channel to collect the Raman band of interest. A flip mirror was used to send the SERS signal to a spectrometer to obtain the spectra corresponding to the image. The narrow band-pass filter was removed from the detection path to obtain the full spectrum. In the elastic channel, neutral density filter sets (ND Filter) with an overall optical density of 7.0 were employed to adjust the elastic scattering intensity to that of the inelastic signal. 
Materials. Carboxylate-modified Fluospheres (diameter: $0.1 \mu \mathrm{m}, 2 \mathrm{wt} \%$ solid) were purchased from Invitrogen. Benzyldimethylhexadecylammonium chloride (BDAC), cetylpyridinium chloride (CPC), gold chloride trihydrate $\left(\mathrm{HAuCl}_{4} \cdot 3 \mathrm{H}_{2} \mathrm{O}, 99.9 \%\right.$ trace metals), silver nitrate $\left(\mathrm{AgNO}_{3}, 99.9999 \%\right)$, ammonium hydroxide, sodium borohydride $\left(\mathrm{NaBH}_{4}\right), \mathrm{L}$-ascorbic acid (99.9\%), and potassium bromide (KBr) were purchased from Sigma-Aldrich. Hexadecyltrimethylammonium bromide (CTAB) and hexadecyltrimethylammonium chloride (CTAC) were purchased from Tokyo Chemical Industry (TCI).

Synthesis of raspberry-like metamolecules (raspberry-MMs). Raspberry-MMs were synthesized by a literature procedure. ${ }^{1}$ First, $100 \mu \mathrm{L}$ of carboxylate-modified Fluosphere solution (diameter: $0.1 \mu \mathrm{m}, 2 \%$ solids) was mixed with $100 \mu \mathrm{L}$ of $0.01 \mathrm{M} \mathrm{Ag}\left(\mathrm{NH}_{3}\right)_{2}{ }^{+}$and left to stand at room temperature for $30 \mathrm{~min}$. Subsequently, $1 \mathrm{~mL}$ of ultrapure water $(18 \Omega)$ was added to the solution and the mixture was centrifuged at $13,500 \mathrm{rpm}$ for $30 \mathrm{~min}$. The supernatant was discarded, and the precipitate was resuspended in $1 \mathrm{~mL}$ of ultrapure water. The same washing step was repeated once more, and the final precipitates were resuspended in $500 \mu \mathrm{L}$ of ultrapure water. Freshly prepared $0.01 \mathrm{M} \mathrm{NaBH}_{4}(100 \mu \mathrm{L})$ solution was quickly added to this solution with vigorous mixing. This solution was stored overnight before use. The final seed solution was washed by centrifugation at 13,500 rpm for $30 \mathrm{~min}$ and resuspended in $1 \mathrm{~mL}$ of ultrapure water. The growth solution was prepared by mixing an aqueous solution of $\operatorname{BDAC}(0.1 \mathrm{M}, 10 \mathrm{~mL}), \mathrm{HAuCl}_{4} \cdot 3 \mathrm{H}_{2} \mathrm{O}(0.01 \mathrm{M}, 421 \mu \mathrm{L}), \mathrm{AgNO}_{3}(0.01 \mathrm{M}, 64 \mu \mathrm{L})$, and ascorbic acid $(0.1 \mathrm{M}, 67 \mu \mathrm{L})$ sequentially. Then, $7 \mu \mathrm{L}$ of seed solution was added to the growth solution with gentle mixing and allowed to stand at $30^{\circ} \mathrm{C}$ for $2 \mathrm{~h}$. After $2 \mathrm{~h}$, the solution was washed by centrifugation at 3,000 rpm for $10 \mathrm{~min}$. The supernatant solution was removed, and the precipitate was resuspended in ultrapure water. 
Synthesis of $\sim 10-\mathbf{n m}$ Au seed nanoparticles. The 10-nm Au nanoparticle solution was prepared using a modified seed-mediated growth method. ${ }^{2}$ First, the seed solution was prepared by mixing an aqueous solution of CTAB $(0.1 \mathrm{M}, 5 \mathrm{~mL})$ and $\mathrm{HAuCl}_{4} \cdot 3 \mathrm{H}_{2} \mathrm{O}(0.01 \mathrm{M}, 125 \mathrm{~mL})$. The freshly prepared ice-cold NaBH4 solution $(0.01 \mathrm{M}, 300 \mu \mathrm{L})$ was quickly injected into the seed solution and vigorously mixed for $1 \mathrm{~min}$. The resulting solution was allowed to stand at $27^{\circ} \mathrm{C}$ for at least $2.5 \mathrm{~h}$. The growth solution was prepared by mixing an aqueous solution of CTAB (0.2 M, $20 \mathrm{~mL})$, ascorbic acid (0.1 M, $15 \mathrm{~mL})$, and seed solution $(250 \mu \mathrm{L})$. Then, 0.5 mM HAuCl${ }_{4} \cdot 3 \mathrm{H}_{2} \mathrm{O} 10 \mathrm{~mL}$ was added to the solution and gently mixed for $30 \mathrm{~s}$. Lastly, the resulting solution was allowed to stand at $27^{\circ} \mathrm{C}$ for $2 \mathrm{~h}$. After $2 \mathrm{~h}$, the solution was centrifuged at 13,500 rpm for $30 \mathrm{~min}$ and washed with $0.1 \mathrm{M} \mathrm{CPC}$ three times.

Synthesis of Au nanocubes. Au nanocubes were prepared using the seed-mediated growth method. $^{3}$ The growth solution was prepared by mixing $\mathrm{CPC}(0.1, \mathrm{M} 50 \mathrm{~mL}), \mathrm{KBr}(0.1 \mathrm{M}, 5$ $\mathrm{mL}), \mathrm{HAuCl}_{4} \cdot 3 \mathrm{H}_{2} \mathrm{O}(0.01 \mathrm{M}, 500 \mu \mathrm{L})$, and ascorbic acid $(0.1 \mathrm{M}, 750 \mu \mathrm{L})$ sequentially. Then, $150 \mu \mathrm{L}$ of CPC-capped 10-nm Au nanoparticle solution (OD: 1) was added to the growth solution with gentle mixing. The solution was allowed to stand at $27{ }^{\circ} \mathrm{C}$ for $2 \mathrm{~h}$. The final product was collected by centrifugation at $6,000 \mathrm{rpm}$ for $10 \mathrm{~min}$ and washing once with ultrapure water.

Characterization. Extinction spectra were measured with an Agilent $8453 \mathrm{UV}-\mathrm{Vis}$ spectrometer. Scanning electron microscope (SEM) images were obtained using JEOL JSM$7610 \mathrm{~F}$ at an accelerating voltage of $15 \mathrm{kV}$. 
Bimodal SERS/Mie scattering measurement. For SERS/Mie scattering imaging, each nanoparticles were deposited on a cover glass, which was then submerged in a solution of a Raman reporter, 4-mercaptobenzoic acid (4-MBA, $1 \mathrm{mM}$ ) for $3 \mathrm{~h}$ to ensure that the variations in the SERS signals originated from distinct plasmonic enhancements at the nanogaps rather than from differences in the number of 4-MBA molecules at the gaps. SERS signals of 4-MBA at $1075 \mathrm{~cm}^{-1}$ were recorded from raspberry-MMs and nanocubes using a $660 \mathrm{~nm}$ excitation wavelength and an acquisition time of $500 \mathrm{~ms}$. This signal corresponds to the most prominent band ascribed to the ring stretch mode of our Raman reporter, and was routed through the narrow-bandpass (NB) filter installed in the inelastic scattering channel, as shown in Figure 1b. From a preliminary experiment with fluorescent nanospheres, the positional coincidence between these two elastic/inelastic optical signals in our optical assembly was determined to be within 1.0 and 1.0 pixels for the $x$ - and $y$-axis, respectively, on the CCD image plane. Herein, a single pixel corresponds to $60 \mathrm{~nm}$ under the overall magnification $(\times 210)$ of our imaging assembly.

\section{Finite-Different Time-Domain (FDTD) modeling}

Numerical simulations were carried out with a commercial FDTD software package (Lumerical FDTD, Lumerical Solutions) to calculate the electrical field distribution for raspberry MMs and nanocube dimers. A plane wave source normally incident onto the dimers was employed. The PS core in the raspberry MMs had a refractive index of 1.5. The refractive index for the Au in the raspberry MMs and nanocubes was obtained from Johnson and Christy. ${ }^{4}$ For the background refractive index, that of water $(n=1.33)$ was used. The raspberry MMs, PS core and Au nanoparticles were composed of diameters of $100 \mathrm{~nm}$ and $36 \mathrm{~nm}$, respectively. The gap between the Au particles was within $2 \mathrm{~nm}$. For the nanocubes, the edge length was $63 \mathrm{~nm}$. The calculated electric field distribution was observed at a wavelength of $660 \mathrm{~nm}$. 


\section{Supplementary Results}

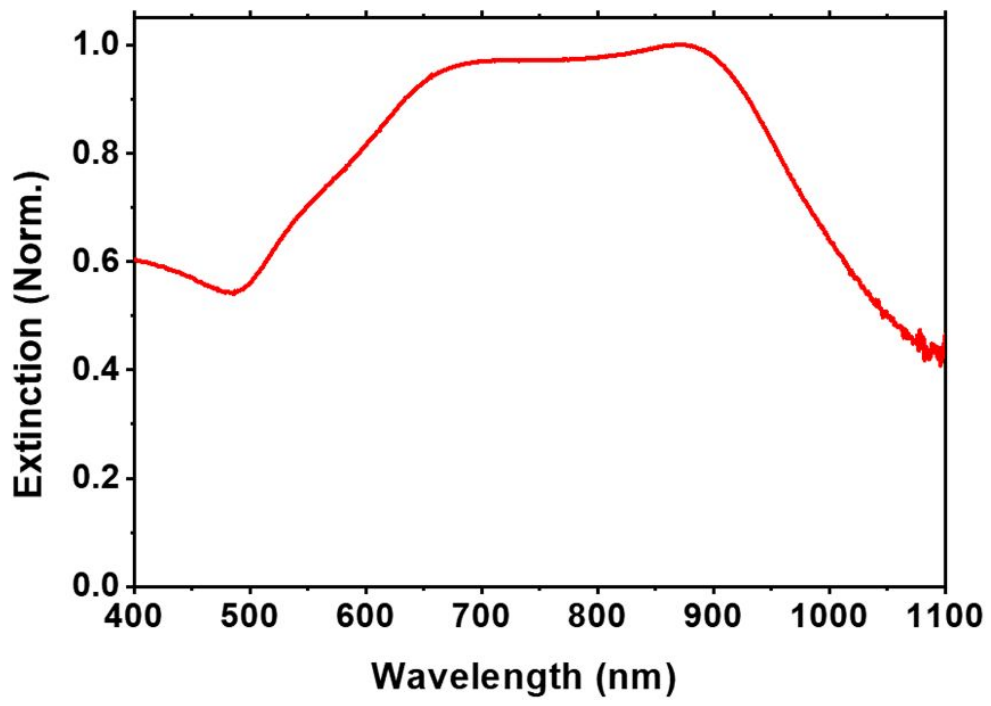

Figure S1. The extinction spectrum of raspberry-MMs. 


\section{SERS}

\section{Mie}

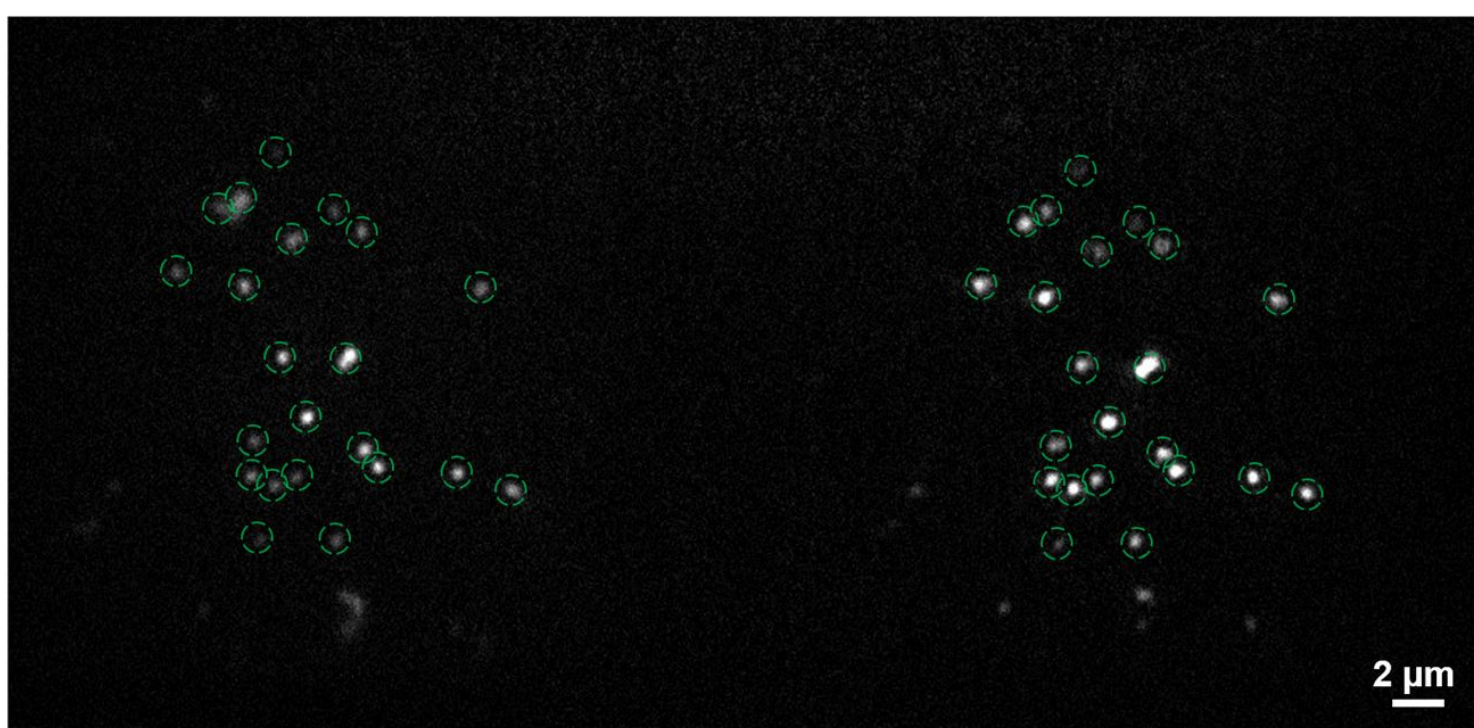

Figure S2. An image of the raspberry-MMs in a chosen detection area. 
a
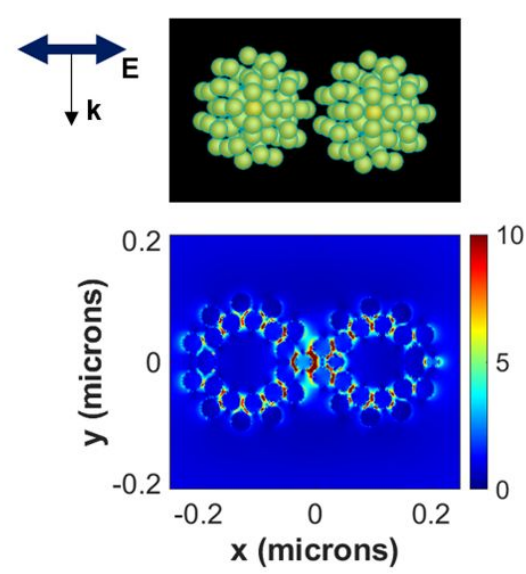

b
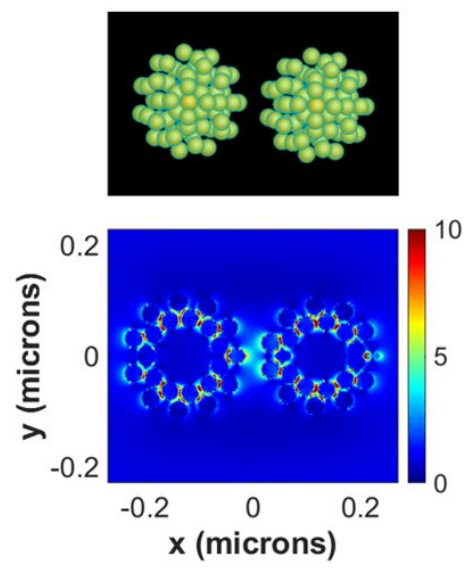
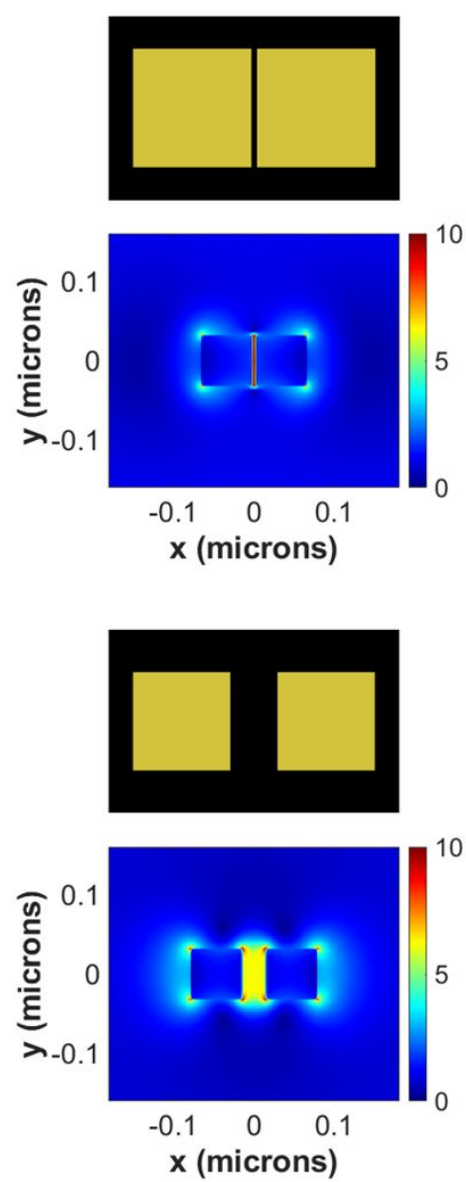
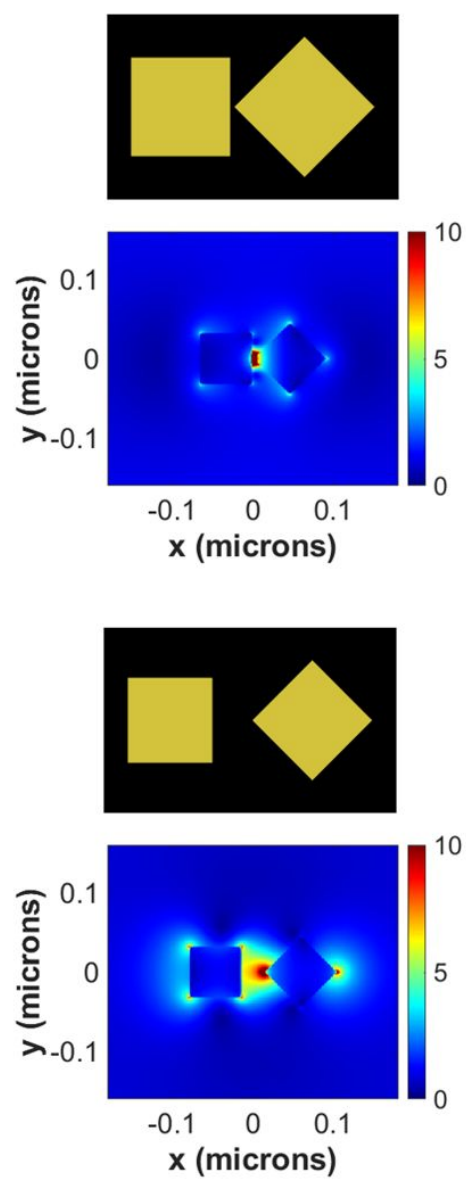

Figure S3. Calculated field-distribution of a pair of (left) raspberry-MMs, (middle) face-toface Au nanocubes, and (right) edge-to-face Au nanocubes, excited by $660 \mathrm{~nm}$-light. The dimer intergap spacing is (a) 3 and (b) $30 \mathrm{~nm}$. 

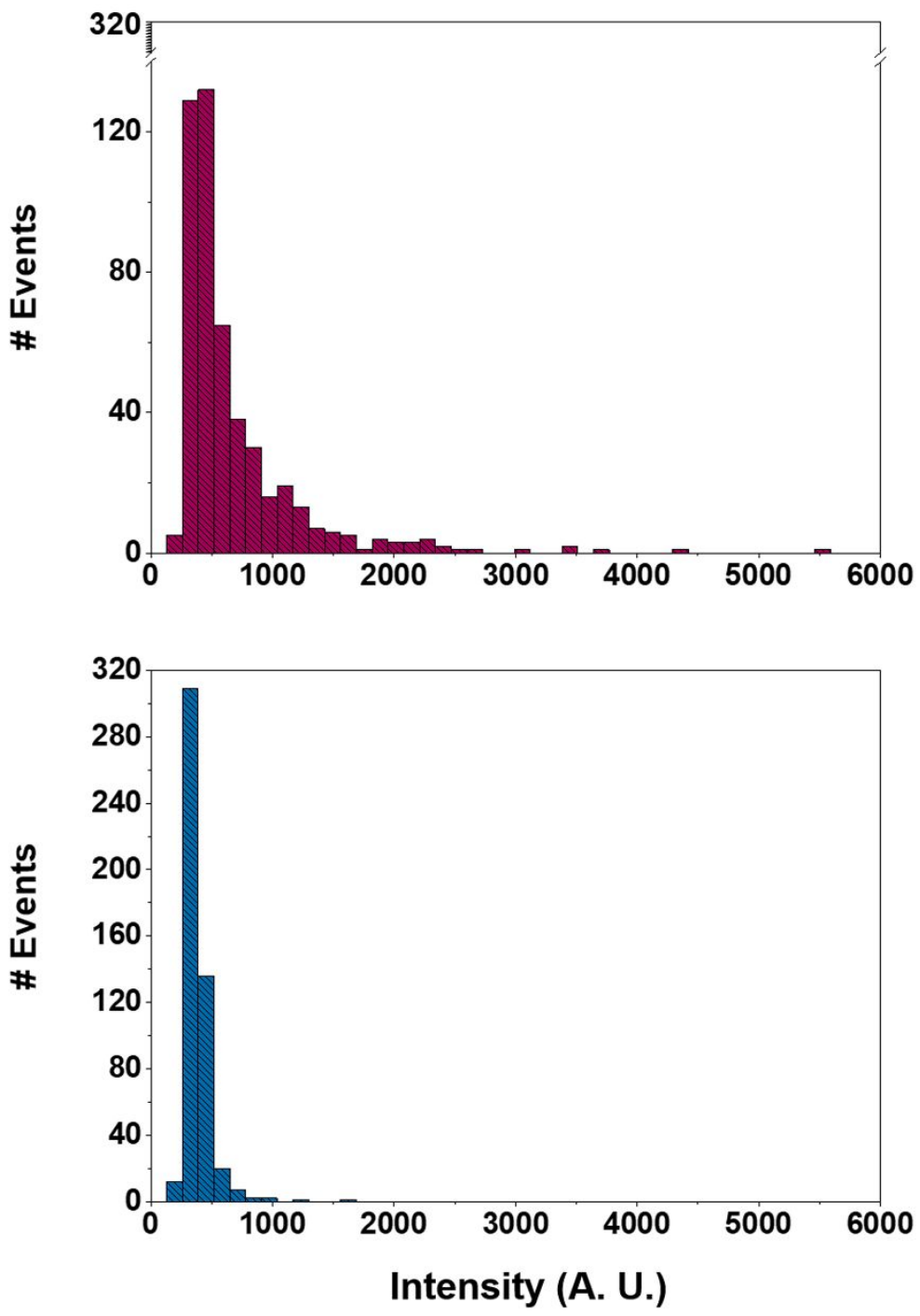

Figure S4. SERS signal distributions obtained from 490 cube (upper) and 492 raspberry-MMs (lower). 
a

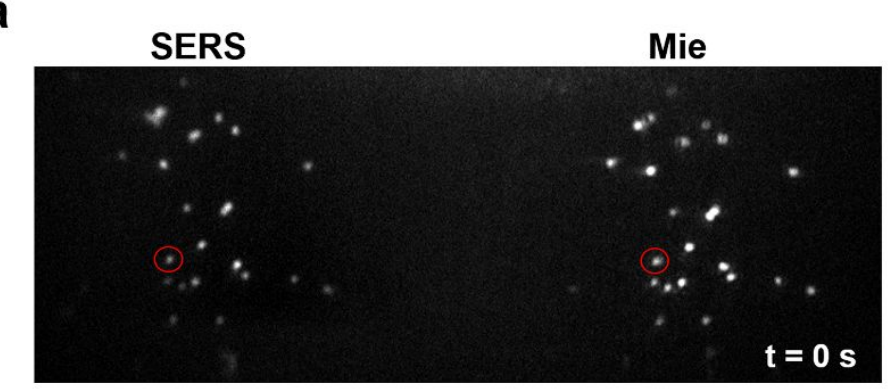

b

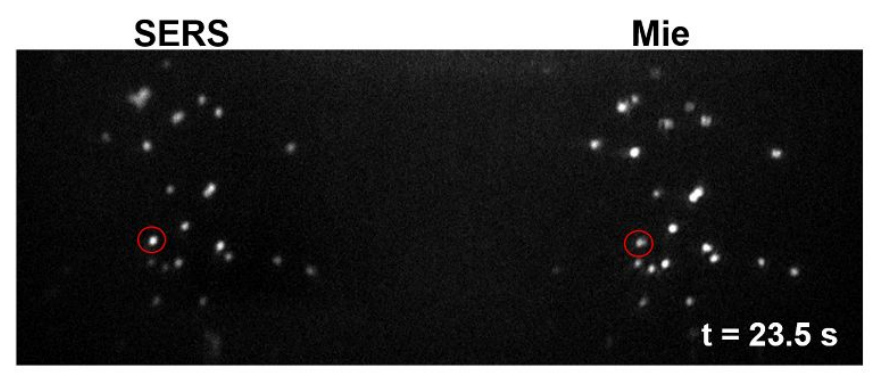

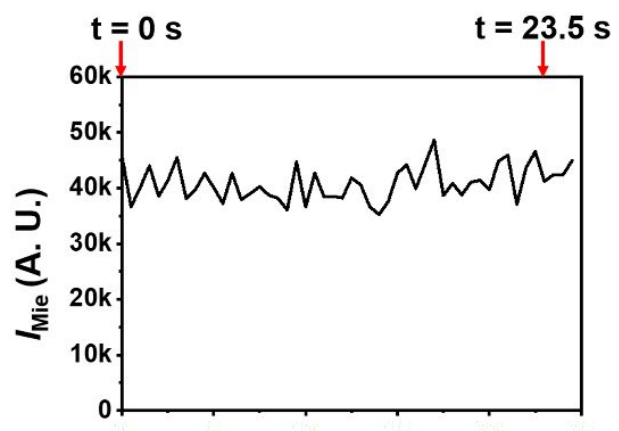

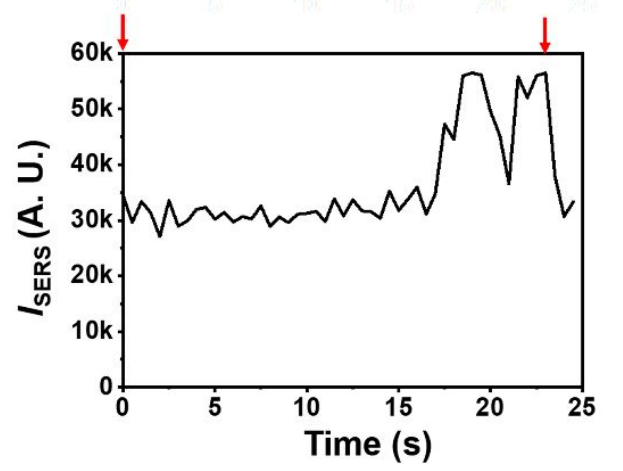

Figure S5. Images (left) and time traces (right) of SERS and Mie scattering signals, chosen at (a) $0 \mathrm{~s}$ and (b) $23.5 \mathrm{~s}$, from raspberry-MMs. 
a
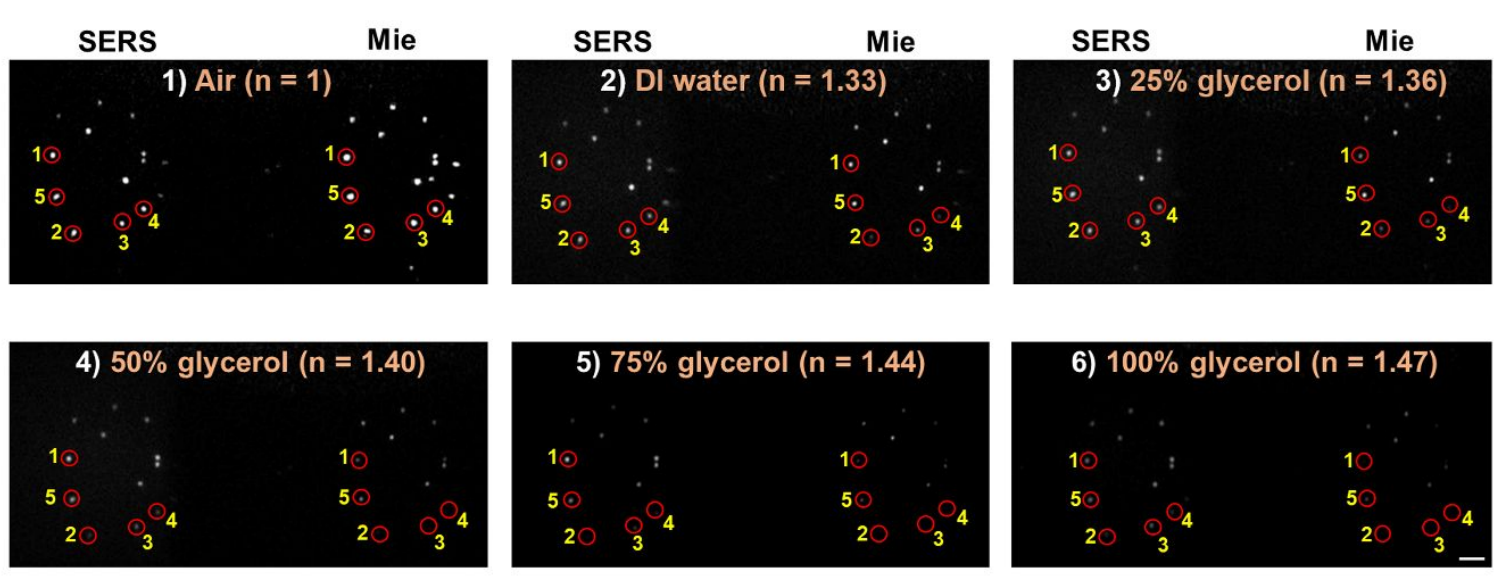

b
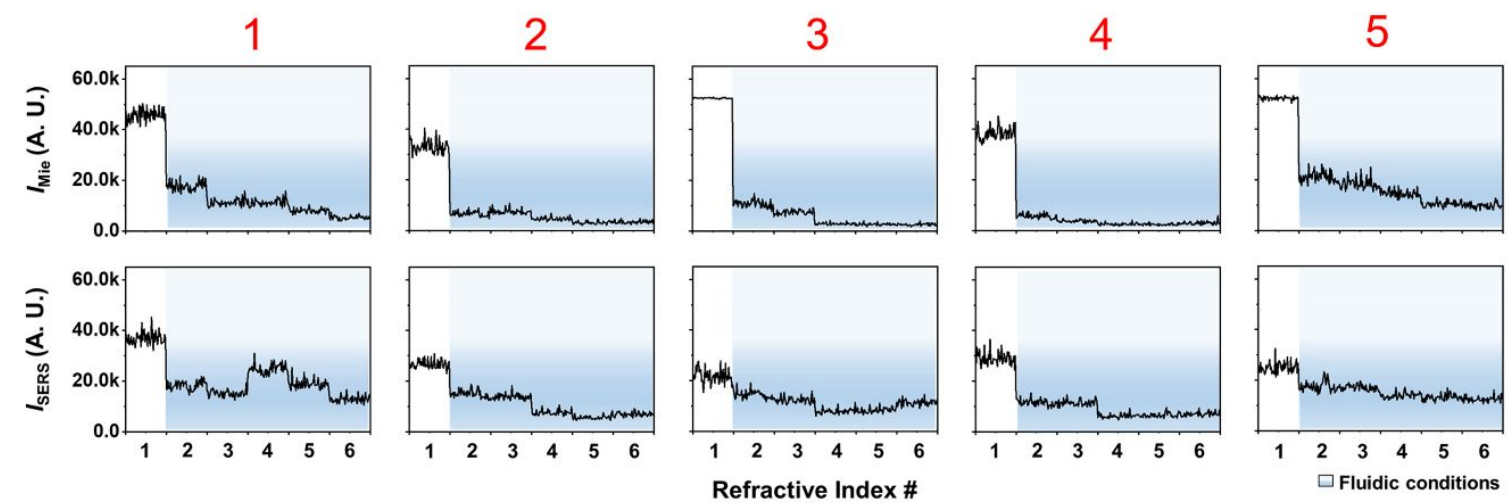

Figure S6. RI dependence of SERS and Mie scattering signals of raspberry-MMs. A scale bar in (a) represents $2 \mu \mathrm{m}$. 


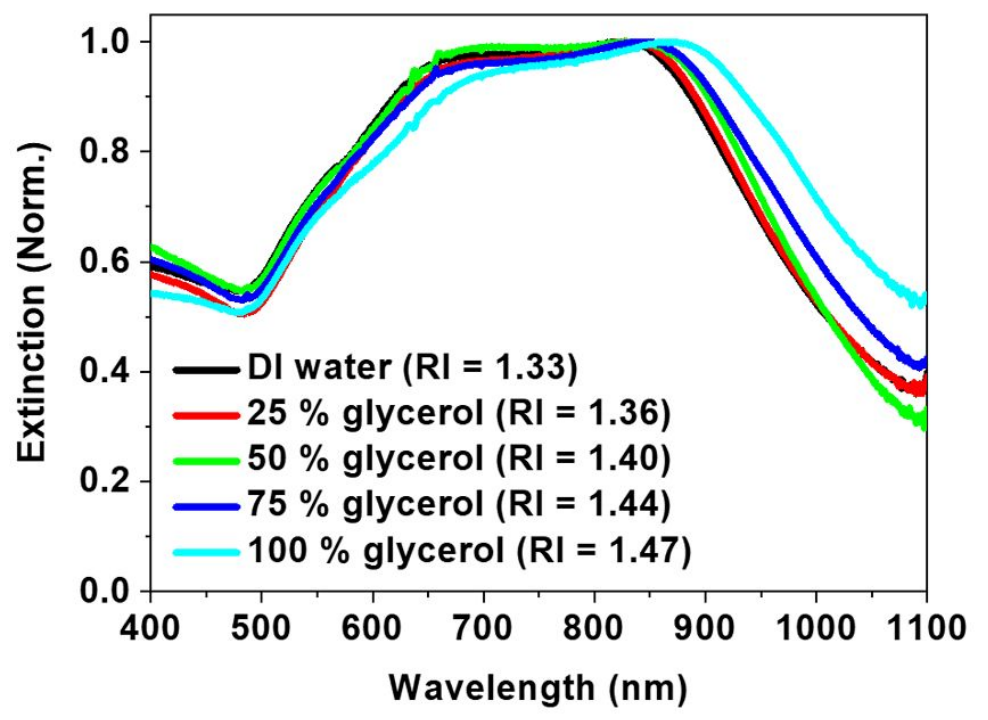

Figure S7. Extinction spectra of raspberry-MMs with varying refractive index (RI) from $\mathrm{n}=1.33$ to 1.47 . The RI values were adjusted by changing the water and glycerol composition of each raspberry-MM solution. 

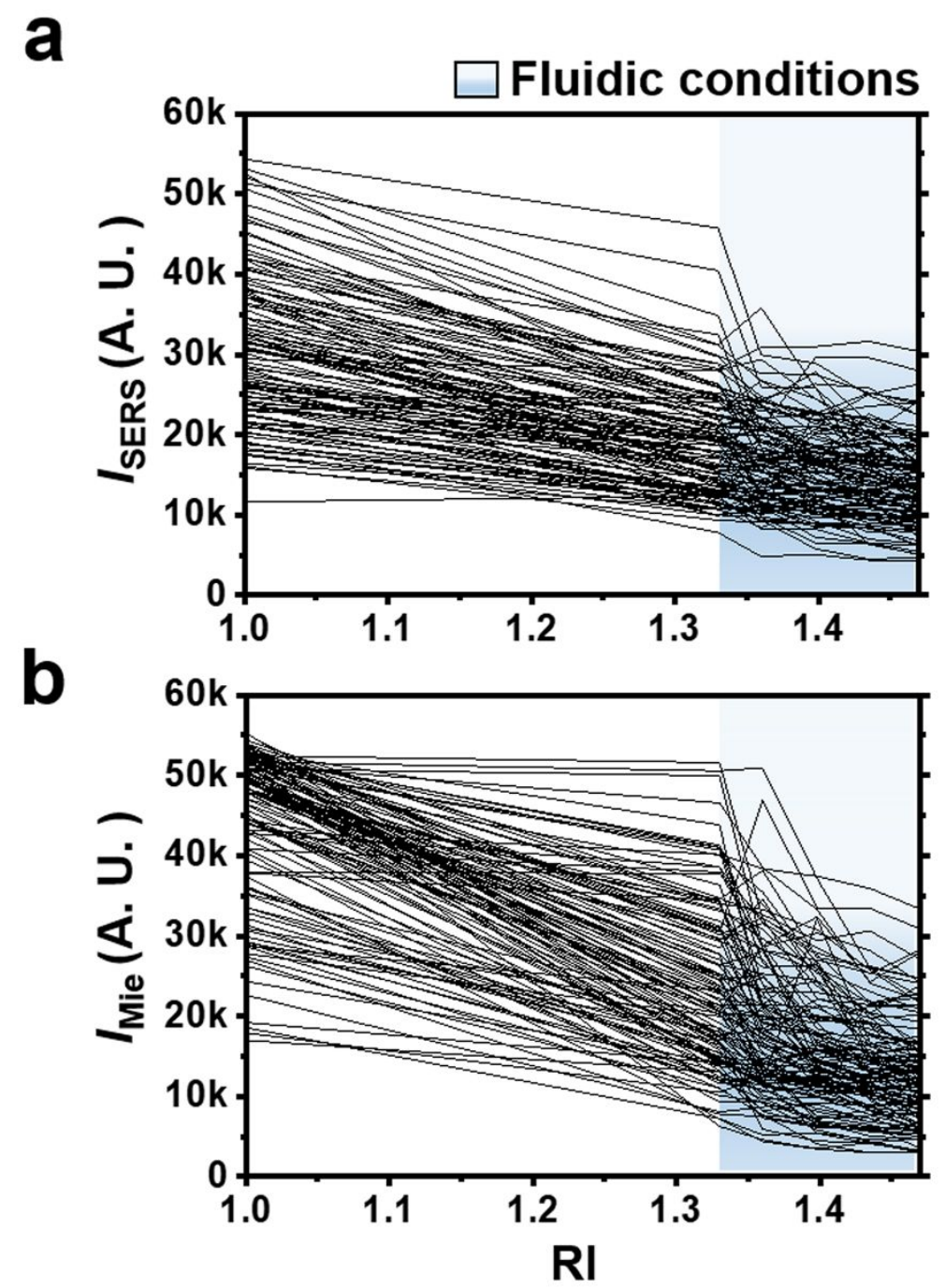

Figure S8. Intensities of SERS (a) and Mie scattering (b) signals from 110 raspberry-MMs tracked over the RI. 

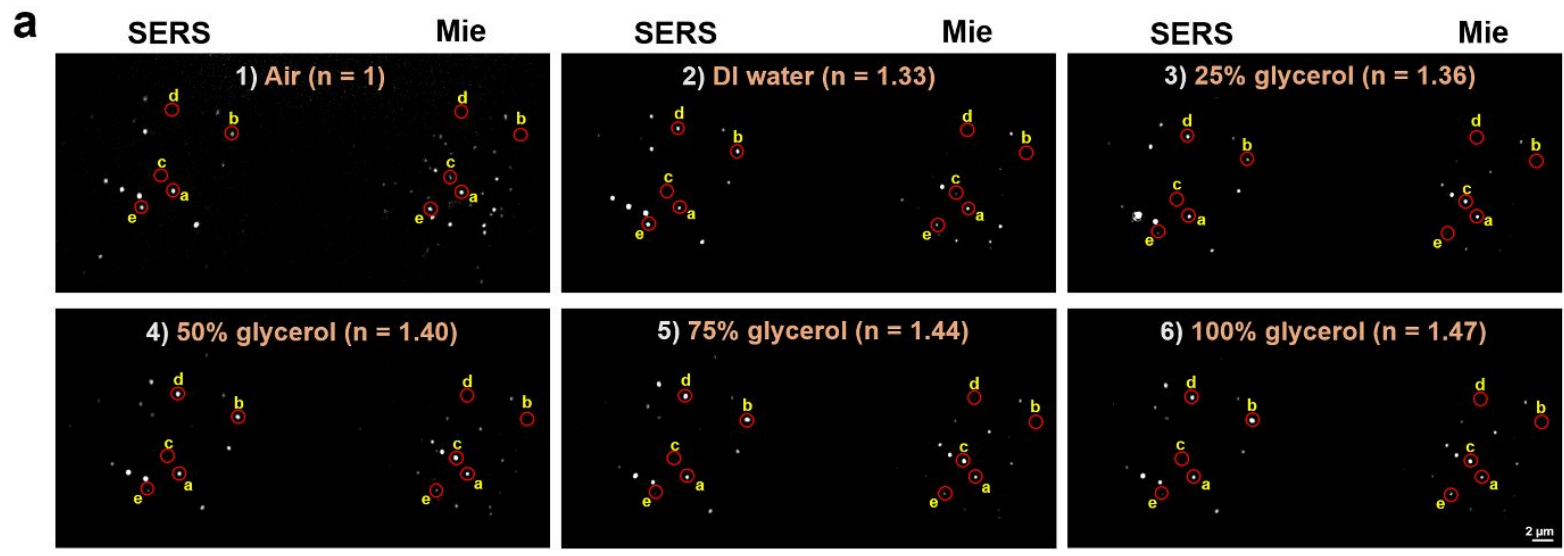

b

b
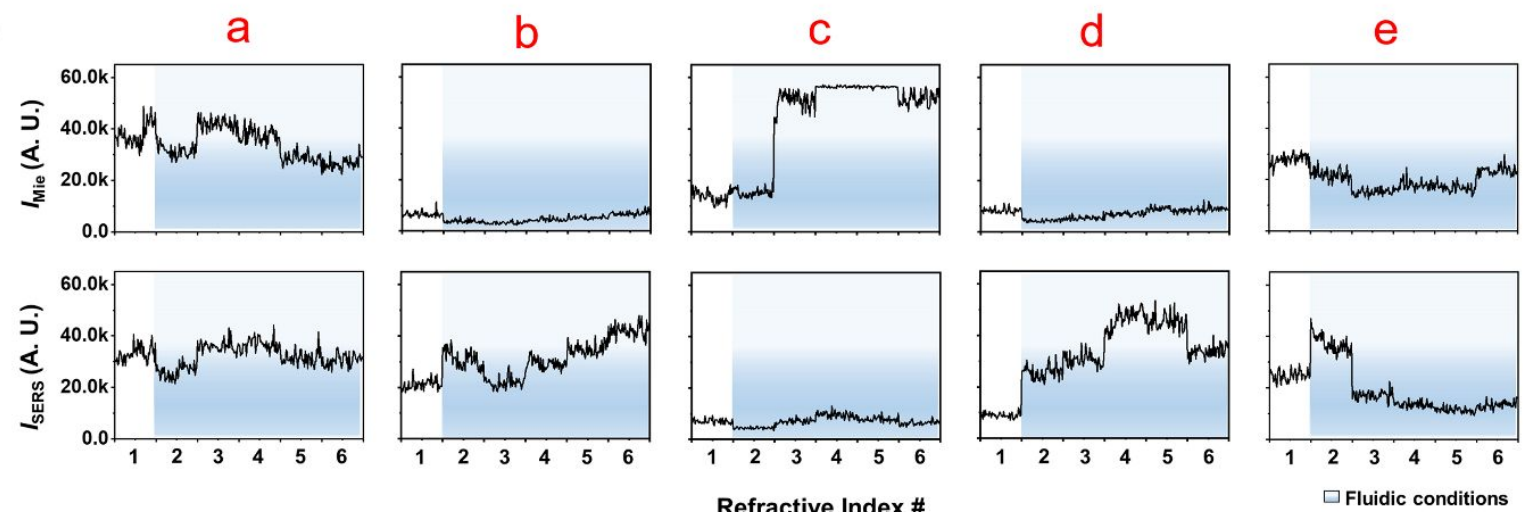

Figure S9. RI dependence of SERS and Mie scattering signals of Au cube particles. (a) Snapshot images of SERS and Mie scattering signals from cube particles immersed in media of varying RI from 1 to 1.47. (b) Mie scattering (upper panel) and SERS (lower panel) intensities from five nanocubes (marked by red circles in (a)) in media of varying RI. Observed heterogeneity is generally expected for asymmetric nanostructures excited by polarized incidence as in this study. 


\section{References}

1. Qian, Z.; Hastings, S. P.; Li, C.; Edward, B.; McGinn, C. K.; Engheta, N.; Fakhraai, Z.; Park, S.-J. Raspberry-like Metamolecules Exhibiting Strong Magnetic Resonances. ACS Nano 2015, 9, 1263-1270.

2. Zheng, Y.; Zhong, X.; Li, Z.; Xia, Y. Successive, Seed-Mediated Growth for the Synthesis of Single-Crystal Gold Nanospheres with Uniform Diameters Controlled in the Range of 5-150 nm. Part. Part. Syst. Char. 2014, 31, 266-273.

3. O’Brien, M. N.; Jones, M. R.; Brown, K. A.; Mirkin, C. A. Universal Noble Metal Nanoparticle Seeds Realized Through Iterative Reductive Growth and Oxidative Dissolution Reactions. J. Am. Chem. Soc. 2014, 136, 7603-7606.

4. Johnson, P. B.; Christy, R.-W. Optical Constants of the Noble Metals. Phys. Rev. B 1972, 6,4370 . 